\title{
$\checkmark$ Feasibility and diagnostic accuracy of Internet-based dynamic telepathology between Uganda and Germany
}

\author{
Dan Wamala*, Achilles Katamba ${ }^{\dagger}$ and Otto Dworak \\ *Department of Pathology, Mulago Hospital and Makerere University College of Health Sciences, Kampala, Uganda; \\ ${ }^{\dagger}$ Clinical Epidemiology and Biostatistics Unit, School of Medicine, Makerere University College of Health Sciences, \\ Kampala, Uganda; ${ }^{\ddagger}$ Institute of Pathology, Fuerth Teaching Hospital of the University of Erlangen, Nuremberg, Germany
}

\begin{abstract}
Summary
We assessed the feasibility and diagnostic accuracy of Internet-based telepathology compared with conventional microscopic examination. A total of 96 cases from the routine workload of the Department of Pathology at the Mulago Hospital in Uganda were examined by robotic telemicroscopy via the Internet at the Fuerth Hospital in Germany. The telepathology diagnoses were compared with those of conventional microscopy. Email and Skype telephony were used to exchange clinical and diagnostic information. The reference diagnosis (gold standard) was established by consensus between two or more experienced pathologists using both conventional microscopy and telemicroscopy; immunohistochemistry was used whenever it was necessary. It took approximately $30 \mathrm{~min}$ for a pathologist to learn to use the telepathology system and 4-25 min to read a case remotely. Internet speed was the main limiting factor. The images were of good quality and the pathologist at the remote site was able to navigate through the slide and change the magnification as necessary. In 92 of the specimens (97\%), the pathologists at the two hospitals agreed exactly about the diagnosis. Agreement overall was moderate $(\mathrm{kappa}=0.39)$. The discordant diagnoses were attributed to factors related to diseases morphologically difficult to diagnose, such as soft tissue sarcomas and primitive tumours. Internet-based conferencing systems offer an inexpensive method of obtaining a primary diagnosis by telepathology and consulting on cases that require subspecialty expertise.
\end{abstract}

\section{Introduction}

Uganda has very few pathologists, and because of this, specialization is usually impossible. It takes an average of 14 days to send a specimen block to Germany and receive the results by email. Telepathology might therefore be a valuable alternative. A pilot trial of static telepathology practised on smears in a rural hospital in Uganda yielded some positive results, as evidenced by a reduced turnaround time, although the quality of the images was poor. ${ }^{1} \mathrm{~A}$ feasibility study of dynamic active telepathology using the Nikon Coolscope was conducted in South Africa over the National Health Laboratory service network. All users assessed the system as a helpful tool which allowed easy access to cases needing a consultation or second opinions. The quality of images was very good. Their experience with the Nikon Coolscope was positive. ${ }^{2}$
Telepathology allows microscopic diagnosis via high-quality images viewed on a video monitor in contrast to conventional light microscopy viewed directly with the naked eye through a microscope. ${ }^{3}$ It allows physical separation between the sites where the specimen is processed and where the diagnosis or consultation is rendered. Dynamic (i.e. realtime) telepathology allows the physician at the remote location to manipulate the slide on the microscope stage at the initiating location, thereby giving him or her control over what is viewed, rather than being shown selected images by the physician at the initiating site. ${ }^{4-6}$ Initial concerns about diagnostic accuracy and image quality have led to improvements in telepathology technology. Images for telepathology can be delivered to the remote site in several ways:

(1) Store-and-forward static imaging; ${ }^{4}$

(2) Dynamic (robotic) imaging;

(3) Hybrid dynamic/static imaging; ${ }^{7}$

(4) Virtual microscopy, a method of posting microscope images on a computer network to allow viewing by people in different locations; ${ }^{8,6}$

(5) Via smart phones, which allow rapid specialist referrals to be made. 
The types of services provided to the remote site include primary diagnosis, consultations with an expert, quality assurance, proficiency testing and education. ${ }^{8-12}$ Although dynamic telepathology has been successfully implemented in several industrialized countries, it does not yet appear to have been used between developing countries and industrialized countries.

The present study was undertaken to assess the feasibility and accuracy of dynamic telepathology between pathologists at the Mulago Hospital in Uganda and the Fuerth Hospital in Germany.

\section{Methods}

Between January 2007 and April 2008, routinely stained sections from surgical pathology specimens from different organs were randomly selected from the routine diagnostic workload of the Mulago Hospital. The sections were analysed by conventional microscopic examination at the Mulago Hospital, and telemicroscopically at the Fuerth Hospital in Germany. Paraffin sections were prepared according to standard procedures. Tissue specimens obtained at surgery were fixed in 10\% formalin and embedded by routine histopathology processing. Four-micrometer sections were cut, stained with haematoxylin and eosin, and mounted. Usually two experienced pathologists at the Mulago Hospital together with remote telepathologists, and where necessary additional tests like immunohistochemistry, established a diagnosis through consensus.

The pathologists at the Mulago Hospital made a diagnosis based on all available clinical information and examination of the microscopic sections. Then the remote pathologist was notified that a case was available for viewing. The remote pathologist was apprised of the clinical information, details of the gross examination of the specimen and then had control of the robotic system to view the slide to establish a diagnosis. Cases were later reviewed to determine the reasons for discordant telepathology diagnosis.

We used a telemicroscopy system (Coolscope, Nikon) in which sections on a computerized digital microscope at the initiating laboratory were remotely controlled by the pathologist at the second laboratory. The Coolscope incorporates a digital microscope, a digital camera and web server capabilities in a single package. Everything, from observation through image capture to data

communications over the network, is operated by a series of simple mouse clicks. Once the glass slide is set, bright-field images can be viewed on the monitor. Focus and brightness can be adjusted automatically or manually. A controller enables operation similar to an actual microscope. The dynamic images of the Coolscope are available to the telepathologist via an Internet browser. The major advantage is that any pathologist with access to the Internet can provide a primary diagnosis or give a second opinion. ${ }^{13-15}$

\section{Feasibility}

The pathologist at the Mulago Hospital placed the slide of interest on the Coolscope and initiated a videoconferencing session with the remote pathologist using Skype. The two pathologists could simultaneously view the same images on their respective computer monitors. Although the system allowed the pathologists to talk to each other as they explored the images on their respective monitor screens, in the present study the service was not used to discuss the pathological diagnosis of the specimens, as this would have biased the remote pathologist.

Assessment of feasibility was done by recording the length of time it took for images to be transmitted (Internet speed), turnaround time (from the time of biopsy until the time the results were ready) and image quality. The image quality was considered good if it was uniform and easily readable, and poor if the image quality did not allow the slide to be confidently diagnosed. Feasibility was assessed as good if the remote pathologist could adjust focus and magnification without difficulty and poor if they could not.

\section{Accuracy}

Light microscopy occasionally assisted by ancillary tests like immunohistochemistry was used as the gold standard for diagnosis. When necessary, tissue blocks were sent to Germany to enable the pathologists to look at cases using light microscopy and immunohistochemistry in order to establish a gold standard. Cases were also discussed at the Mulago Hospital using a light microscope in the local slide seminars. The diagnosis eventually agreed on was the gold standard.

\section{Analysis}

Data were entered into a management program (EpiData version 3.1, EpiData Association, Odense, Denmark) and analysed using a statistical package (SPSS version 11 for Windows). We compared agreement on the pathological diagnoses of the specimens between pathologists in the two hospitals using the kappa statistic.

\section{Results}

A total of 96 consecutive specimens representing disease conditions from 16 different organ systems were selected for the study (see Table 1).

It took about 30 min for the pathologists to learn how to operate the system and become comfortable with manipulating the robotic connection. The pathologists thought that the system was easy to operate. It took 4-25 min to perform the telepathology procedure depending on the Internet speed and complexity of the case. The quality of the images as reported by the pathologist at the remote centre was good, and the remote 
Table 1 Anatomical site of the specimens studied $(n=96)$

\begin{tabular}{lccc}
\hline Site of specimen & Malignant & Benign & Total \\
\hline Skin & 1 & 5 & 6 \\
Bone and joints & 4 & 3 & 7 \\
Breast & 5 & 2 & 7 \\
Endocrine system & 0 & 3 & 3 \\
Haematopoietic and lymphatic system & 10 & 1 & 11 \\
Head and neck & 4 & 2 & 6 \\
Major and minor salivary glands & 2 & 4 & 6 \\
Urinary system & 5 & 1 & 6 \\
Male reproductive system & 2 & 3 & 5 \\
Female reproductive system & 4 & 6 & 10 \\
Peritoneum, mediastinum & 1 & 0 & 1 \\
Soft tissue & 5 & 12 & 17 \\
Central nervous system & 1 & 1 & 2 \\
Alimentary canal and associated organs & 6 & 3 & 9 \\
Total & 50 & 46 & 96 \\
\hline
\end{tabular}

pathologist could navigate through the slide and change magnification with ease.

The pathologists at both hospitals independently read all specimens. In 92 of the specimens (97\%), the pathologists at the two hospitals agreed exactly about the diagnosis. The agreement overall was moderate (kappa $=0.39)$. The discordant diagnoses were attributed to factors related to diseases morphologically difficult to diagnose, such as soft tissue sarcomas and primitive tumours (Table 2).

Some slides were of poor quality (poor smear preparation, tissue sectioning and staining), which hampered general interpretation as well as preventing the auto focus capability of the microscope from working. In some cases ancillary tests which were critical to diagnosis were not available, e.g. special stains to identify organisms, or stains to identify tumour type. The smaller the specimen was, the easier it was to look at all the material on the slide and make a diagnosis. Another frequent problem limiting the diagnostic capability of the consultant pathologist was the absence of supporting clinical data, laboratory data and molecular studies.

\section{Discussion}

The present study demonstrates that telepathology using the Internet-based Coolscope can offer a quick and

Table 2 Discordant diagnoses in 4 of the 96 specimens

\begin{tabular}{lll}
\hline Site of specimen & Pathologist 1 (Uganda) & $\begin{array}{l}\text { Pathologist } 2 \\
\text { (Germany) }\end{array}$ \\
\hline $\begin{array}{c}\text { Soft tissue from right thigh } \\
\text { muscle }\end{array}$ & Rhabdomyosarcoma & Liposarcoma \\
$\begin{array}{l}\text { Left kidney } \\
\begin{array}{c}\text { Soft tissue from right } \\
\text { scapular }\end{array}\end{array}$ & $\begin{array}{l}\text { Nephroblastoma } \\
\text { Alveolar rhabdomyosarcoma } \\
\begin{array}{c}\text { Soft tissue in } \\
\text { supraclavicular area }\end{array}\end{array}$ & $\begin{array}{l}\text { Rhabdoid tumour } \\
\text { Malignant } \\
\text { melanoma }\end{array}$ \\
\hline
\end{tabular}

relatively accurate method for pathology diagnosis in a developing country. In 92 out of 96 cases the pathologists at the Mulago Hospital were in agreement with the diagnosis made by the pathologist at the remote site in Germany.

On average, it took $10 \mathrm{~min}$ to perform the telepathology procedure. Dynamic telepathology and videoconferencing were performed with relative ease using the Coolscope and Skype. The Skype software also allowed the user to transfer files and send text messages. The findings from the present study reveal that diagnostic accuracy is similar to that obtained by light microscopy. The discordant diagnoses were attributed to factors related to diseases that were morphologically difficult to diagnose.

The Internet has been used in previous telepathology studies, ${ }^{14,16-18}$ but most studies have used static images because these are generally of higher visual quality and require lower transmission bandwidth than do dynamic images transmitted in realtime. However, static-image telepathology is hindered by the possibility of sampling error related to inadequate or inappropriate selection of microscopic fields for imaging by the referring pathologist. Dynamic telepathology systems using robot microscopes are potentially more useful, because they allow the remote pathologist to visualize all materials on a particular slide using variable magnification and thus achieve higher diagnostic accuracy. Videoconferencing systems linked by point-to-point connections have been used for telepathology in other laboratories. ${ }^{18,19}$ Studies done by Leong et al. in Oxford showed that robotic telepathology is accurate for primary diagnosis in pulmonary histopathology, but modifications in laboratory protocols and telepathology technology would be necessary to make telepathology usable in a busy referral practice. ${ }^{20}$

Pathology videoconferencing systems, a TV phone system and the Internet have been used to transmit still and realtime images between the Armed Forces Institute of Pathology in Washington, DC and the National Cancer Institute in Tokyo. Twenty cases of varying histology were transmitted, and the authors concluded that the system provided an effective method for exchanging pathology information. ${ }^{8,19}$

Telepathology can be used for remote primary diagnosis, referral to a specialist pathologist, teaching, presentation of post-mortem or microscopic findings, quality assurance image circulation and feedback, and consensus diagnosis for pathological review in clinical trials.

Dynamic telepathology is a sophisticated technique allowing the viewing pathologist to have remote control of a microscope at a distant site. Slides can be viewed in their entirety at various magnifications derived from a high resolution scan of the slide and the images displayed on the Internet browser are a complete representation of the actual glass slide. Thus telepathology can provide a frozen section service and second opinion consultation in sites where there is only limited availability of a pathologist. ${ }^{21}$ The main advantage of using the Internet is that it is accessible to everybody, so that any pathologist may become a consultant in telepathology. This contrasts with previous 
closed systems, which offered communication between fixed workstations. This technique has now received extensive attention and widespread acceptance. However, its diagnostic efficiency and accuracy have not previously been evaluated in Uganda. The development of telepathology for consultation is of relevance to Uganda, not only to support consultations with super-specialized centres outside the country but also between the local pathology laboratories.

It has also been shown previously ${ }^{21}$ that diagnosis using the Coolscope robotic microscope becomes equivalent to conventional microscopy after several years of use, so it is possible that our diagnostic accuracy could improve from that which we identified in the present study.

\section{Limitations}

The main problems with telepathology in our study were pre-analytical in nature, and included poorly sectioned, stained and mounted sections, and lack of ancillary tests like special stains and immunohistochemistry, especially for poorly differentiated tumours such as sarcomas and primitive tumours. Lymphomas are very difficult to analyse without immunohistochemistry. Careful attention to pre-analytic factors with improved technical training and use of special stains like immunohistochemistry would probably improve diagnosis by telepathology. Another frequently observed problem was the absence of supporting clinical data, laboratory data and molecular studies, and this limited the diagnostic ability of the consultant pathologist. When available, such data would also improve diagnostic accuracy in telepathology.

Other limitations were related to the technology itself. Although in industrialized countries the Internet is almost ubiquitous and relatively cheap, in the developing world its use is still expensive, it is unreliable and only low bandwidth is available. These problems are likely to be overcome in the next few years.

In summary, the results of our study show that the use of telepathology for primary diagnosis and consultation in a wide variety of surgical pathology specimens is feasible, with good concordance between pathologists in a developing and an industrialized country. Internet-based conferencing systems offer an inexpensive method of obtaining a primary diagnosis by telepathology and consulting on cases that require subspecialty expertise. In both instances, turnaround time is greatly improved, benefiting patients.
Acknowledgements: We thank Professor O Ferrer-Roca whose bibliography was a great asset in our work and in the preparation of this paper.

\section{References}

1 Isyagi M. Static telepathology and biopsy report waiting time: report of a pilot experience in Africa. See http://www.iweee.org/presentations/2010/ isyagi-IWEEE-static-telepathology.pdf (last checked 24 November 2010)

2 Banach L, Stepien A, Schneider J, Wichrzycka-Lancaster E. Dynamic active telepathology over National Health Laboratory service network, South Africa: feasibility study using Nikon Coolscope. Diagn Pathol 2008;3 (Suppl. 1):S3

3 Cross SS, Dennis T, Start RD. Telepathology: current status and future prospects in diagnostic histopathology. Histopathology 2002;41: 91-109

4 Weinstein RS, Bhattacharyya AK, Graham AR, Davis JR. Telepathology: a ten-year progress report. Hum Pathol 1997;28:1-7

5 Kayser K, Szymas J, Weinstein RS. Telepathology. Telecommunications and electronic education and publication in pathology. Heidelberg, Germany: Springer-Verlag, 1999;1-186

6 Weinstein RS, Descour MR, Liang C, et al. Telepathology overview: from concept to implementation. Hum Pathol 2001;32:1283-99

7 Zhou J, Hogarth MA, Walters RF, Green R, Nesbitt TS. Hybrid system for telepathology. Hum Pathol 2000;31:829-33

8 Walter GF. Teleneuropathology: a means to improve the correctness of neuropathological diagnoses in clinical practice. Crit Rev Neurosurg 1999;9:1-11

9 Wootton R. The possible use of telemedicine in developing countries. J Telemed Telecare 1997;3:23-6

10 Vassallo DJ, Swinfen P, Swinfen R, Wootton R. Experience with a low-cost telemedicine system in three developing countries. $J$ Telemed Telecare 2001;7(Suppl. 1):56-8

11 Eusebi V, Foschini L, Erde S, Rosai J. Transcontinental consults in surgical pathology via the Internet. Hum Pathol 1997;28:13-6

12 Della Mea V, Roberto V, Conti A, di Gaspero L, Beltrami CA. Internet agents for telemedicine services. Med Inform Internet Med 1999;24:181-8

13 Swinfen P, Swinfen R, Youngberry K, Wootton R. A review of the first year's experience with an automatic message-routing system for low-cost telemedicine. J Telemed Telecare 2003;9(Suppl. 2):63-5

14 Della Mea V, Puglisi F, Forti S, et al. Expert pathology consultation through the Internet: melanoma versus benign melanocytic tumours. J Telemed Telecare 1997;3(Suppl. 1):17-9

15 Horbinski C, Fine JL, Medina-Flores R, Yagi Y, Wiley CA. Telepathology for intraoperative neuropathologic consultations at an academic medical center: a 5-year report. J Neuropathol Exp Neurol 2007;66:750-9

16 Della Mea V, Forti S, Puglisi F, et al. Telepathology using Internet multimedia electronic mail: remote consultation on gastrointestinal pathology. J Telemed Telecare 1996;2:28-34

17 Della Mea V, Puglisi F, Bonzanini M, et al. Fine-needle aspiration cytology of the breast: a preliminary report on telepathology through Internet multimedia electronic mail. Mod Pathol 1997;10:636-41

18 Edworthy SM. Telemedicine in developing countries. BMJ 2001;323:524-5

19 Petersen I, Wolf G, Roth K, Schlüns K. Telepathology by the Internet. J Pathol 2000;191:8-14

20 Leong FJ, Nicholson AG, McGee JO. Robotic telepathology: efficacy and usability in pulmonary pathology. J Pathol 2002;197:211-7

21 Fraser HS, Jazayeri D, Bannach L, Szolovits P, McGrath SJ. TeleMedMail: free software to facilitate telemedicine in developing countries. Stud Health Technol Inform. 2001;84:815-9 